\title{
A Review on Sensors for Real-time Monitoring and Control Systems on Machining and Surface Finishing Processes
}

\author{
Tomi Wijaya ${ }^{1}$, Wahyu Caesarendra ${ }^{1}$, Tegoeh Tjahjowidodo ${ }^{2,}{ }^{*}$, Bobby $K$ Pappachan $^{1}$, Arthur \\ Wee $^{3}$, Muhammad Izzat Roslan ${ }^{3}$ \\ ${ }^{1}$ Rolls-Royce @ NTU Corporate Laboratory, Singapore 639798 \\ ${ }^{2}$ School of Mechanical and Aerospace Engineering, Nanyang Technological University. Singapore \\ 639798 \\ ${ }^{2}$ Rolls-Royce Singapore, Applied Technology Group, Singapore 639798
}

\begin{abstract}
One of the key components in real-time monitoring and control on machining and surface finishing processes are sensors. The advances of such system have triggered interesting questions on sensor selection that act as the fundamental before starting a project. This paper is made to review and answer the questions surrounding sensor selection. The paper first explains on the type of sensors commonly used in practice for realtime monitoring and control systems. After which, the paper discusses on how often the sensors are used on several machining and surface finishing processes and what are the reasons for the sensor selection. Thereafter, a review on the type features commonly analysed through these sensors is discussed. The paper expects reader would decide better upon selecting sensors and has a better direction in their project. Thus the paper works to guide reader to improve based on what has been completed before.
\end{abstract}

\section{Introduction}

The development of manufacturing industry nowadays is pushed to its limit to fulfil the demand of market. The market requires better quality products, more variability, shorter product life cycle, reduced product cost, and globally competitive products [1].

Machining and surface finishing processes develops in the direction of immediate integration with real-time monitoring and control systems to allow users to monitor and control the events happening during machining and surface finishing.

The selection of sensors is one of the fundamental building block for real-time monitoring. This paper will discuss on the sensors used in other real-time monitoring and control projects to better inform reader on suitable sensor application for their project.

\footnotetext{
* Corresponding author: ttegoeh@ntu.edu.sg
} 


\section{Methodology}

The development of real-time monitoring and control systems on machining and surface finishing processes consists of several procedural steps as presented in the figure below

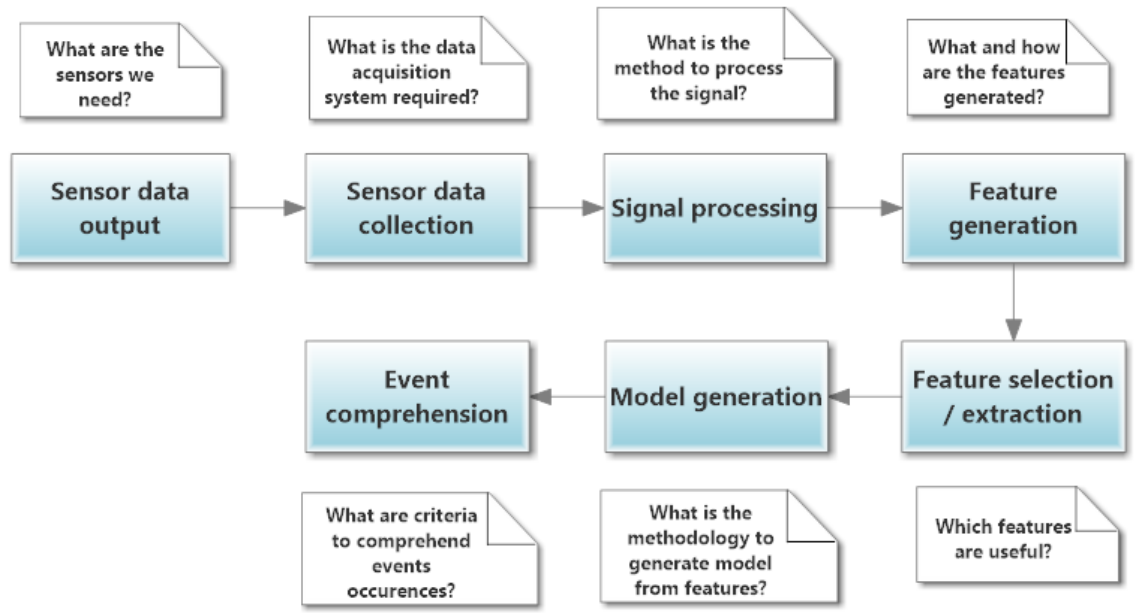

Fig 1. Development methodology

The methodology mentioned above is the generic methodology often used in real-time monitoring and control systems which uses sensors for events interpretation. The scope of this paper covers on "Sensors data output", "Feature generation/calculation" and "Feature selection/extraction". Sensors selection determine the sensors data output. Meanwhile, "Feature generation/calculation" and "Feature selection/extraction" develop by understanding the available features and how these features fit into the application. These three steps is covered considering that the result varies if the three steps were to change.

\section{Selection of sensors type system}

Commonly, the type of sensors used for real-time monitoring and control for machining and surface finishing processes are acoustic emission sensor, accelerometer, force sensor, and dynamometer/power meter. These 4 sensors are common because they measure the variables involved directly in the processes. Acoustic emission sensor measures the elastic wave emitted from the processes. Accelerometer measures the acceleration of tools or work coupon during the process and detects anomalies or signatures for each event. Force sensor measures the force applied on the interaction between work coupon and tool. Dynamometer / power meter measures the difference in the power behaviour upon different events during the machining or finishing processes. The summary of frequently used sensors for real-time monitoring and control systems in manufacturing processes is presented Table 1.

\subsection{Acoustic emission sensor}

According to Table 1, the frequently used sensors for manufacturing processes is $\mathrm{AE}$ sensor. Caesarendra et.al. [2] mentioned that AE-based method can be applied in wide range frequencies generated from two processes i.e. (i) impacting, and (ii) friction and rubbing. In manufacturing, the impact between work coupon and tools generates instantaneous AE signal. Due to the abrasive process, a transient elastic wave generated by the contact interaction of the work coupon and abrasive tool. Moreover, raw AE signal can 
be analysed with two general techniques namely: (1) AE-hit parameters and (2) AE signal processing. The AE-hit parameters are calculated from the built-in AE software. The example of AE-hit parameters are amplitude, RMS, energy, counts, events, ASL and AE burst. Aside from AE-hit parameters, some signal processing techniques can be applied for AE signal such as short-time Fourier transform (STFT), wavelet package decompositionHilbert Huang transform (WPD-HHT), and Wigner-Ville distribution.

Inasaki [3] used AE sensor on turning, cutting and grinding processes and suggested that $\mathrm{AE}$ sensor to be complimented with other sensor such as force sensor due to $\mathrm{AE}$ sensor's high sensitivity to the process state.

Y.B Guo et.al. [4] concluded that AE RMS, frequency and count rate can be used to distinguish surface damage, tool wear and surface roughness. In addition, AE frequency strongly correlate to surface finish quality. Surface damage increases the peak of AE amplitude and its corresponding frequency.

Table 1. Frequently used sensors for real-time monitoring and control systems

\begin{tabular}{|l|c|c|c|c|}
\hline \multirow{4}{*}{$\begin{array}{c}\text { Surface Finishing \& } \\
\text { Machining Process }\end{array}$} & \multicolumn{4}{|c|}{ Sensor System } \\
\cline { 2 - 5 } & $\begin{array}{c}\text { Acoustic } \\
\text { Emission }\end{array}$ & Accelerometer & Force sensor & Power meter \\
\cline { 2 - 5 } & & & & \\
& & & & \\
\hline 1.Drilling & {$[5-7]$} & {$[7]$} & {$[6-9]$} & {$[7,8]$} \\
\hline 2.Lapping & {$[7,10]$} & {$[7,10]$} & {$[7,9,10]$} & {$[7,10]$} \\
\hline 3. Grinding & {$[3,7,10]$} & {$[7,10]$} & {$[7,9,10]$} & {$[7,10]$} \\
\hline 4.Milling & {$[3,7,11,12]$} & {$[7,13]$} & {$[7-9,11,13]$} & {$[7,8,13]$} \\
\hline 5.Broaching & {$[7,14]$} & {$[7,14]$} & {$[7,9,14]$} & {$[7]$} \\
\hline 6.Turning & {$[4,7,10,15]$} & {$[7,10,15,16]$} & {$[7-10,15]$} & {$[7,8,10,15]$} \\
\hline \multicolumn{1}{|c|}{ Total } & 10 & 6 & 9 & 5 \\
\hline
\end{tabular}

\subsection{Accelerometer}

Accelerometer is more frequently used than $\mathrm{AE}$ in vibration analysis of rolling element bearing [2].This is because of its well-developed data acquisition and analysis tools [17]

Accelerometer can be placed on the work coupon or on the tool and measures the vibration on which it is attached to. The frame of reference for accelerometer is always on the attached surface. If accelerometer is attached to work coupon, it measures vibration when tool is touching the work coupon. However when it is attached to tool, the accelerometer measures vibration from the tool when the tool is in operation. Frame of reference will help to differentiate events occurrence from the accelerometer signal.

Salgado, D.R. et.al [16] used SSA decomposition and LS-SVM to predict the quality of surface roughness. In addition, Salgado, D.R. et.al [16] recommended to use three axis accelerometer for better prediction result

\subsection{Force sensor}

External force sensors can be applied in two different place. First is on the tool. Once the tool applies force on work coupon, the reacting force to the force sensor will give out the sensor reading. The second one is on work coupon. The sensor detects the reacting force only if the force application area is on the sensor placement. 
Force sensor can be applied by integrating it into a direct driven motor spindle using piezo electric force sensor rings. Byrne et.al [6] mentioned that by integrating the force sensor into the motor spindle, it enables the reading of non-process related forces. The higher frequency content received from the sensor reveals spindle signature for monitoring.

M.B. Jun et.al. [9] used Kistler force ring sensor, an 8 uniaxial piezoelectric force sensor. They concluded that the force sensor affected the spindle dynamics slightly through the time-varying temperature differentials, which to be solved using temperature control on the spindle. The signal signatures is similar to Byrne et.al [6] conclusion. There were two signatures, one belong to the spindle internal dynamics and the other to machining. M.B. Jun et.al. compare the machining signature produced by the force sensor and the dynamometer and found similarities in the calibration model resulted

\subsection{Power meter / dynamometer}

Dynamometer measures the power output of an engine by measuring torque and the rotational speed. When events occur out of the routine, dynamometer indicates power difference. Two placements are possible for dynamometer, embedded and non-embedded.

Axinte, D et. al. [8] uses Kistler dynamometer to measure the spindle power signal in turning (9257B4), milling (9272) and drilling (9272) processes. The aim is for tool condition monitoring. In milling, the force sensor was sensitive enough to detect milling cutter tooth chipped. In turning and drilling, dynamometer helps in detecting such event.

\section{Frequency specifications of sensors}

In order to obtain more obvious signatures related to the surface finishing stage and quality, the sensor signal processing can be performed in either time-domain or frequency domain. In machining processes, often a rotating tool is employed for material removal and hence frequency domain analysis can help to extract meaningful information from the incoming sensor signal. It is imperative in such applications to identify the frequency range of interest and select suitable sensors accordingly. To extract the frequency range of interest, the typical method used is spectral analysis. Figure below shows commonly used sensors for process monitoring in machining classified based on operating frequency range and from this figure it can be deduced that $\mathrm{AE}$ and accelerometer are more commonly used

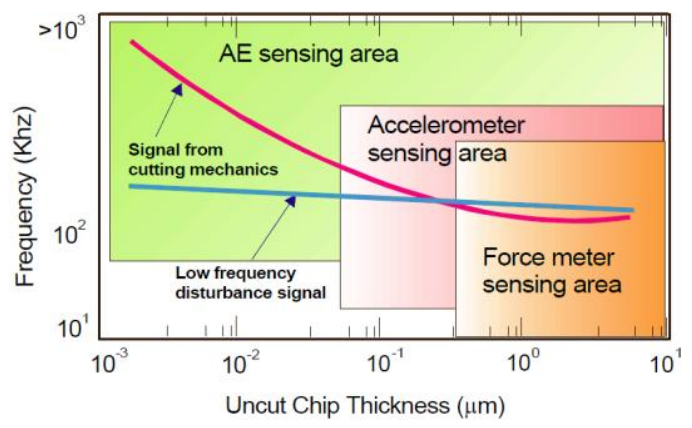

Fig 2. Sensor classification based on operating frequency range and material removal rate [18]

This information aids in identifying and subsequently isolating potential noise that contains no direct information regarding the process. 


\section{Sensor signal features generation and extraction}

In frequency analysis, Fast Fourier Transform (FFT) is the most commonly used method for its ability to identify the dominant frequency event. However, FFT performs effectively in stationary periodic signals and it is less effective for non-stationary signals that arise from time-dependent events. There are a number of features generation in frequency analysis of vibration signal as has been reviewed and discussed in [17]. It is worth noting that the review features are applied in vibration signal of rolling element bearings that may not suitable for other application. The FFT has been applied in manufacturing field as a feature or descriptor that known as signal harmonic (single H) [7]. The following is the summary studies that presented the frequency feature analysis in machining processes.

Table 2. Frequently domain features applied for machining processes

\begin{tabular}{cccc}
\hline Signal & \multicolumn{3}{c}{ Frequency-domain Feature } \\
\cline { 2 - 4 } & Single H & PSD & H ratios \\
\hline Acoustic emission & {$[10,49]$} & {$[9,49,32,36]$} & {$[50]$} \\
Vibration & {$[10,27,16,30,5,49,50]$} & {$[27,49]$} & {$[23]$} \\
Force & {$[10,14,9,49,50]$} & {$[14,5,49]$} & {$[\mathrm{NA}]$} \\
Power & {$[\mathrm{NA}]$} & {$[\mathrm{NA}]$} & {$[\mathrm{NA}]$} \\
\hline
\end{tabular}

The signal features can be categorised into three domains, time domain, frequency domain, and wavelet domain. The features for each domain is summed up in table 3 below.

Table 3. Signal domains and features for sensors data analysis

\begin{tabular}{|l|l|}
\hline Time & $\begin{array}{l}\text { RMS, Peak, Mean Value, Standard Deviation, Skewness, Kurtosis, } \\
\text { Variance, Autoregressive (AR) models, Time-domain averaging (TDA) }\end{array}$ \\
\hline Frequency & Harmonic Ratios, Single harmonic, Power spectral density (PSD) \\
\hline Wavelet & RMS, Peak \\
\hline
\end{tabular}

\section{Conclusion}

The current trend on real-time monitoring and control systems on machining and surface finishing covers different approaches in methodology and type of sensors. This paper has completed the initial steps to align the methodology and given the resources on sensors that have been used before in other researches. The key points that the paper has achieved are:

- Laying down the generic methodology, concluded from the methodologies that have been used before in other projects.

- Gathered the resources of sensors used in other project according to the machining process it is used on. In addition, how the sensors can be used in the project. This includes the possible placement, advantages and disadvantages of the sensor.

- Hinted on frequency analysis on different sensors that leads to different frequency specification per sensor

- Elaborated on the features commonly used in real-time monitoring and control systems, including the equation definition of this feature. In addition, the signal extraction and example of FFT in the signal processing. 


\section{References}

1. S. Y. Liang, R. L. Hecker, R. G. Landers, J. Manuf. Sci. Eng. 126 (2004).

2. W. Caesarendra, B. Kosasih, A. K. Tieu, H. Zhu, C. A. Moodie, Q. Zhu, Mech. Syst. Signal Process. 72 (2016).

3. I. Inasaki, Ultrasonics, 36 (1998).

4. Y. B. Guo, S. C. Ammula, Int. J. Mach. Tools Manuf. 45 (2005).

5. S. H. Lee, D. A. Dornfeld, J. Manuf. Sci. Eng.123 (1999).

6. G. Byrne, G. E. O’Donnell, CIRP Ann. - Manuf. Technol. 56 (2007).

7. J. V. Abellan-Nebot, F. Romero Subirón, Int. J. Adv. Manuf. Technol. 47 (2010).

8. D. Axinte, N. Gindy, Int. J. Prod. Res. 42 (2004).

9. M. B. Jun, O. Burak Ozdoganlar, R. E. DeVor, S. G. Kapoor, A. Kirchheim, G. Schaffner, Int. J. Mach. Tools Manuf. 42 (2002).

10. D. A. Dornfeld, Y. Lee, A. Chang, Int. J. Adv. Manuf. Technol. 21 (2003).

11. I. Marinescu, D. Axinte, Int. J. Mach. Tools Manuf. 49 (2009).

12. I. Marinescu, D. A. Axinte, Int. J. Mach. Tools Manuf. 48 (2008).

13. J. C. Chen, B. Huang, Int. J. Adv. Manuf. Technol. 21 (2003).

14. D. A. Axinte, N. Gindy, K. Fox, I. Unanue, Int. J. Mach. Tools Manuf. 44 (2004).

15. R. Teti, K. Jemielniak, G. O'Donnell, D. Dornfeld, CIRP Ann. - Manuf. Technol. 59 (2010).

16. D. R. Salgado, F. J. Alonso, I. Cambero, A. Marcelo, Int. J. Adv. Manuf. Technol. 43 (2009).

17. W. Caesarendra, PhD Thesis, University of Wollongong (2015).

18. D. Dornfeld, Y. Lee, A. Chang, Int. J. Adv. Manuf. Technol. 21 (2003). 